\title{
EL DEBATE PARLAMENTARIO SOBRE CONCILIACIÓN FAMILIAR Y LABORAL EN ESPAÑA: ANÁLISIS DE UNA DÉCADA DE INICIATIVAS ${ }^{1}$
}

\author{
Parliamentary debate on reconciling work and family in Spain: \\ analysis of a decade of initiatives
}

\author{
MARTA MARTÍN LLAGUNO² \\ CRISTINA GUIRAO MIRÓN ${ }^{3}$
}

\section{Resumen}

Este estudio realiza un análisis de la presencia del problema de la conciliación familiar y laboral en la agenda parlamentaria del Congreso de los Diputados en la VI, VII y VIII legislatura (19962008). Después de recopilar y contabilizar todas las iniciativas con menciones al tema, se narra la historia política de la cuestión. A continuación, y para valorar su importancia relativa, por meses, años y legislaturas, se computa la razón entre las iniciativas con menciones al conflicto familiar y laboral y el resto de iniciativas. Además, y con el fin de determinar los promotores del tema, se calcular la probabilidad de haberlo mencionado siendo diputado o diputada y siendo gobierno u oposición («odds ratio» [OR] intervalo de confianza [IC] del 95\% y significación estadística [método Mantel-Haenszel]). Se concluye que en España, en la VI y VII legislatura, se genera tímidamente un ciclo de atención política sobre el conflicto familiar y laboral y su solución (la conciliación), que se reenfoca totalmente en la VIII hacia «la conciliación para la igualdad» (momento de más visibilidad parlamentaria del tema). A partir de 2008, el interés parlamentario decae.

Palabras clave: Conciliación familiar y laboral, políticas públicas, iniciativas parlamentarias, toma de decisiones.

\begin{abstract}
This study describes the formulation and decision-making process related to the Spanish Parliament on the reconciliation between work and family. In this article, all the parliamentary initiatives by Deputies on this issue in the VI, VII and VIII Legislatures (1996-2008) are content analysed. After reporting the treatment of the subject, the ratio of work and family reconciliation initiatives compared to other issues is calculated by months, years and legislatures. An analysis is also done of who proposes reconciliation initiatives, whether they belong to the government or the opposition, or if they are male or female («odds ratio» [OR] confidence interval [CI] of $95 \%$ and statistically significant [MantelHaenszel method)]. We conclude that in Spain, in the VI and VII legislatures, a cycle of political attention on the difficulty of the reconciliation between work and family began. During the VIII legislature, the concept was refocused on «conciliation for equality» (and was the moment of the highest parliamentary visibility on the issue). However, from 2008 on, parliamentary interest in this issue declines.
\end{abstract}

Key Words: Work Life balance, public policy, parliamentary initiatives, decisionmaking

Recibido: 07/02/2012 Revisado: 13/04/2012 Aceptado: 08/05/2012 $\quad$ Publicado: 02/11/2012

1. Este artículo forma parte del proyecto I+D: «La construcción social del problema del conflicto familia-trabajo. Análisis mediático, político y de la realidad» que ha sido financiado por el Instituto de la Mujer en su convocatoria 2007/2010. Marta Martín Llaguno fue IP y Cristina Guirao investigadora.

2. Marta Martín Llaguno. Dto. Comunicación Audiovisual y Psicología Social. Universidad de Alicante. Edificio de CC. Sociales. Dcho. 20b. Universidad de Alicante. Tél: 965903869. Marta.martin@ua.es

3. Cristina Guirao Mirón. Dep. Sociología y Política Social. Universidad de Murcia. 


\section{Introducción}

Si bien existe una definición consensuada para el «conflicto» familiar-laboral (Martín Llaguno, Beléndez Vázquez y Hernández Ruiz, 2007), no sucede lo mismo con la «conciliación». El concepto, entendido de múltiples maneras en las políticas públicas (Ballester, 2011), queda pendiente de delimitar y operacionalizar. El disenso terminológico, que implica un debate conceptual, se inicia con la reconciliación (Hoschild, 1989), presentada como la estrategia para solventar la doble jornada en el Programa Comunitario de Acción Social de 1974 (Ballester, 2011: 20). Transformada en conciliación en España, debido a su carga «económico empresarial» ha sido sustituida por corresponsabilidad en algunas esferas. Al margen de matices, en general, las de conciliación, «cobijan políticas públicas y privadas y tendencias de opinión diversas entre sí que conforman un debate de interés en Europa /.../ En este debate se superponen perspectivas temáticas distintas: la primera, la igualdad entre hombres y mujeres /.../ La segunda, la Estrategia Europea de empleo, y muy en particular, el incremento de la tasa de empleo femenina. La tercera, el reto demográfico que plantea el envejecimiento de la sociedad» (Cabeza, 2010: 46). No exentas de posiciones opuestas (Flaquer, 2000; Bustelo y Peterson, 2005; Iglesias de Ussel y Meil Landwerlin, 2001) estas políticas persiguen crear condiciones para que las responsabilidades familiares y laborales se cumplan en forma óptima, con una relación más armónica entre el trabajo remunerado/no remunerado. En consecuencia, deberían fomentan la equidad. A caballo entre la protección de la familia y la promoción de la igualdad, también se vinculan con el derecho laboral, el fiscal o, recientemente, con regulaciones a favor de nuevos usos del tiempo.

España, entre 1999 y 2009, construye todo un marco legal que traduce la preocupación por la conciliación. Este marco, impelido por Europa, no es sino el resultado de un debate conceptual y político que tiene lugar en las cámaras constituyentes. En efecto, la elaboración de iniciativas parlamentarias es la expresión más genuina de la formulación de políticas. Por esta razón, el estudio de las disertaciones sostenidas en el Parlamento ayuda a explicar la definición de un tema ante la sociedad, las dimensiones que se han querido solucionar y los medios de los que, para ello, se ha dispuesto. El interés del debate parlamentario está, como afirma Feliu-Albaladejo (2011), en primer lugar, en «el progreso de las ideas desde el momento de su introducción hasta que se confirma la política» (Renton y Macintosh, 2007: 2), o la policymemory $y$, en segundo lugar, en la observación del comportamiento de los actores que condicionan el resultado de la acción. El estudio empírico de los discursos parlamentarios facilita la comprensión de la construcción de los problemas 
sociales y permite la evaluación del comportamiento deliberativo del legislador (Feliu-Albaladejo, 2011: 1). Para abordarlo, se pueden hacer aproximaciones más constructivistas, y/o más cuantitativas.

Resulta atractivo indagar sobre las concepciones (latentes) que encierran algunos conceptos y categorías (manifiestas) en los textos parlamentarios y estudiar las repercusiones que estas suposiciones conllevan. También, y sirviéndonos de análisis de contenido, es valioso entender cuándo, y cómo, un hecho políticamente irrelevante se convierte en un momento dado en una cuestión de interés legislativo.

Al primer respecto, la construcción social del problema de la conciliación laboral y familiar que nos ocupa ya ha sido parcialmente analizada. Así, entre 2003 y 2006, el grupo MAGEEQ trabajó los encuadres de la «igualdad de género» en diversos discursos (Lombardo y Meier, 2006; Bustelo y Lombardo, 2007). Además de concluir su variabilidad entre textos (leyes, planes, preguntas...) y países (España, Grecia, Austria, Holanda, Hungría y Eslovenia), el equipo detectó en España, desde finales del siglo pasado, un proceso emergente de formulación política (ausente en la mayor parte de países de la UE) que enmarcaba la conciliación en el contexto de la igualdad.

Al segundo respecto, pese a que, como afirman Asián y Rodríguez (2010) en los últimos quince años las políticas de conciliación se han convertido en un objeto de estudio predilecto en Ciencias Sociales, la evolución del interés político por esta cuestión no se ha analizado cuantitativamente. Así, la «parlamentometría» se viene utilizando para revisar sistemáticamente la toma de decisiones en esta esfera en cuestiones relacionadas con género -violencia machista (Vives-Cases et al., 2006), interrupción voluntaria del embarazo (Cambronero-Saiz et al., 2007) o la inmigración (Agudelo-Suarez et al, 2009)- pero no existen estudios centrados en la evolución en el del tratamiento del conflicto-conciliación trabajo familia en las cámaras.

El Congreso de los Diputados ofrece un marco único para el estudio de la construcción política de este tema. No sólo porque es la entidad ante la que el gobierno ha de responder de su gestión sino porque, en el caso de la conciliación, ha sido el principal escenario desde el que se han regulado las condiciones al respecto para toda la ciudadanía española. Además, por la accesibilidad de información, esta cámara brinda excelentes oportunidades para la sistematización de los discursos sobre el tema. En este contexto, el objetivo de este trabajo es observar la conciliación familiar y laboral como tema de agenda parlamentaria durante las tres legislaturas de mayor prodigalidad legislativa sobre la cuestión: la VI, VII y la VIII.

De manera más concreta se pretende, 
1. Computar la frecuencia relativa y la evolución de la aparición del asunto en los textos del Congreso de los Diputados para valorar su importancia,

2. Reconstruir el relato político de la conciliación;

3. Delimitar los promotores y algunas características del discurso parlamentario sobre el tema.

La finalidad del trabajo es ayudar a comprender mejor cuándo, cómo y de la mano de quién, la conciliación familiar y laboral ha cobrado relevancia política en nuestro país.

\section{Métodos}

Para acometer nuestros objetivos, se han recopilado todas las iniciativas parlamentarias en las que han aparecido los términos conciliación/conflicto de la vida familiar y laboral en la VI Legislatura (marzo 1996-abril 2000), VII Legislatura (abril 2000-abril 2004) y VIII Legislatura (abril 2004-abril 2008). Además, se han recogido todas las iniciativas de estos 12 años. Para ello, se ha consultado la página web del Congreso de los Diputados, que constituye una base de datos útil para el análisis de políticas públicas, por su capacidad de aglutinar información de interés para la realización de estudios retrospectivos (cuenta con datos desde el período constitucional establecido tras la dictadura franquista), de fácil acceso, transparente y aceptablemente actualizada ${ }^{4}$. Se han calculado las tasas computando la razón entre las iniciativas que mencionan el conflicto/la conciliación y el total de iniciativas por meses, años y por Legislaturas.

Una vez acopiados los documentos con las menciones, se han codificado de acuerdo a un protocolo que recoge información sobre las coordenadas del texto (año, mes y legislatura de publicación, órgano parlamentario donde tiene lugar la sesión, comisión encargada tipo de iniciativa), sobre las voces que lo han promovido (autor/a, partido político, sexo...) y sobre la definición del conflicto o la conciliación laboral y familiar que hace. Con base en este protocolo se ha realizado un estudio descriptivo basado en frecuencias absolutas y relativas de las variables.

Para determinar las probabilidades que han tenido los hombres frente a las mujeres, el gobierno frente a la oposición y unos partidos sobre otros de mencionar el tema en la esfera parlamentaria se han calculado «odds ratio» (OR), sus intervalos de confianza (IC) del 95\% y los valores de significación

4. http://www.congreso.es 
estadística (método de Mantel-Haenszel). Los mismos cálculos se han realizado para establecer las posibilidades que un partido $u$ otro o un sexo u otro han tenido de tratar determinadas dimensiones específicas del tema. En todos los casos se utilizó el SPSS-11.5.

\section{Resultados}

En los 145 meses explorados se produjeron 319.312 iniciativas parlamentarias, de las que 149 fueron específicas de conciliación familiar y laboral.

\subsection{La frecuencia relativa de la aparición de este tema en la agenda parlamentaria}

En número absoluto de intervenciones la presencia de la conciliación en la agenda parlamentaria resulta muy irregular hasta 2000, pese a la entrada en vigor de la Ley en el 1999. La explicación posible es la urgencia del Gobierno español por sacar esta norma, sin un gran debate parlamentario previo. La obligada transposición al ordenamiento jurídico español de dos directivas europeas $^{5}$, la primera sobre salud y seguridad en el trabajo de la mujer embarazada, que haya dado a luz o en periodo de lactancia; la segunda sobre el permiso parental, que estable el disfrute indistinto entre hombres y mujeres de los permisos parentales hace que la Ley de conciliación se presente abruptamente.

De hecho, no es hasta 2001 cuando las intervenciones sobre el conflicto y la conciliación familiar y laboral comienzan a aumentar, a partir de 7, hasta septuplicarse en 2006 (cuando alcanza la cota máxima de 55 iniciativas). Desde ese momento empiezan a descender hasta casi desaparecer en el primer trimestre de 2008. Los picos máximos absolutos por meses se producen en febrero y junio de 2006 (20 y 11 iniciativas respectivamente). (Ver gráfico ${ }^{\circ} 1$ : tasa de iniciativas sobre conciliación. Por meses).

Observando las tasas, o la presencia relativa del tema por legislatura, es en la VIII (gobernada por el PSOE) cuando la conciliación acapara el interés parlamentario con más fuerza, alcanzando un máximo de 0,68 iniciativas por cada 1000. (Ver gráfico ${ }^{\circ} 2$ : Tasa de iniciativas sobre conciliación por legislaturas VI, VII y VIII).

Por años, en la primera década se observa una tendencia creciente que alcanza su cima en 2006 - con una tasa de 1,02 por 1000-, y que empieza a caer

5. Directivas del Consejo 92/85/CEE, de 19 de octubre y 96/34/CE, del Consejo de 3 de junio. Ley de conciliación 39/1999, BOE nº 266. Sábado 6 de noviembre de 1999. 
en picado en 2007, cerrando un ciclo de interés parlamentario (ver Gráfico $\mathrm{n}^{\circ} 3$ : Tasa de iniciativas sobre conciliación por años).

En cuanto a las tasas por meses destaca la presencia del tema en abril de 2004 (10,6 por 1000), que supone el pico máximo y es el momento en el que con poca actividad parlamentaria, se proponen varias modificaciones a la Ley de conciliación a cuenta del permiso de paternidad; y el año 2006, en general y en especial febrero: 3,77 por 1000 , abril: 1,29 por 1000 , mayo: 1,44 por 1000 y junio: 2,09 por 1000, que son los meses de 2006 en los que comparecen los expertos en comisión (ver Gráfico 4: Presencia del tema por meses. VI, VII, VIII legislatura).

\subsection{Relato del debate político sobre la conciliación}

El discurso parlamentario sobre la conciliación familiar y laboral se inicia en la sexta legislatura, con el PP al frente, a partir de preguntas sueltas al Gobierno sobre la financiación de programas para promover la conciliación a través de la atención primaria en distintas autonomías y provincias. En febrero de 99 se presentan las líneas básicas del Anteproyecto de Ley de conciliación de la vida familiar y laboral, preparado por el Ministerio de Trabajo y Asuntos Sociales, que es aprobado en mayo del 1999. Este anteproyecto, como se puede observar en la gráfica, no acapara demasiada atención de sus señorías.

La séptima legislatura mantiene la estela de la discusión política sobre el tema de manos del Gobierno, que, en junio de 2000, hace una Proposición no de Ley relativa a la incorporación en los trabajos del principio de igualdad de derechos y oportunidades, así como del derecho a la conciliación, para la adaptación de la normativa europea. Seis meses después, plantea otra Proposición relativa a la convocatoria de una Conferencia Sectorial monográfica sobre conciliación de la vida familiar y laboral para promover un Programa Nacional de guarderías y escuelas infantiles, en colaboración con las Comunidades Autónomas, programa que se aprueba. En el ejercicio de su labor de control, el PSOE pregunta reiteradamente por los permisos por maternidad y cuidado de hijos en aplicación de la Ley de conciliación de la vida familiar y laboral y por la valoración de esta normativa. Y entre junio y noviembre de 2001, insatisfechos con la adaptación final que se ha hecho de las directivas europeas, el grupo socialista y el grupo mixto hacen sendas proposiciones para la introducción de los permisos de paternidad en los supuestos de parto y para la ampliación de derechos. Ambas son rechazadas.

El año 2002 se inicia con preguntas del control al Gobierno sobre los «efectos» de la Ley de conciliación: en concreto sobre las incorporaciones de mujeres al mundo laboral realizadas desde su entrada en vigor. También 


\section{Gráfico 1.}

Tasa de iniciativas sobre conciliación. Por meses

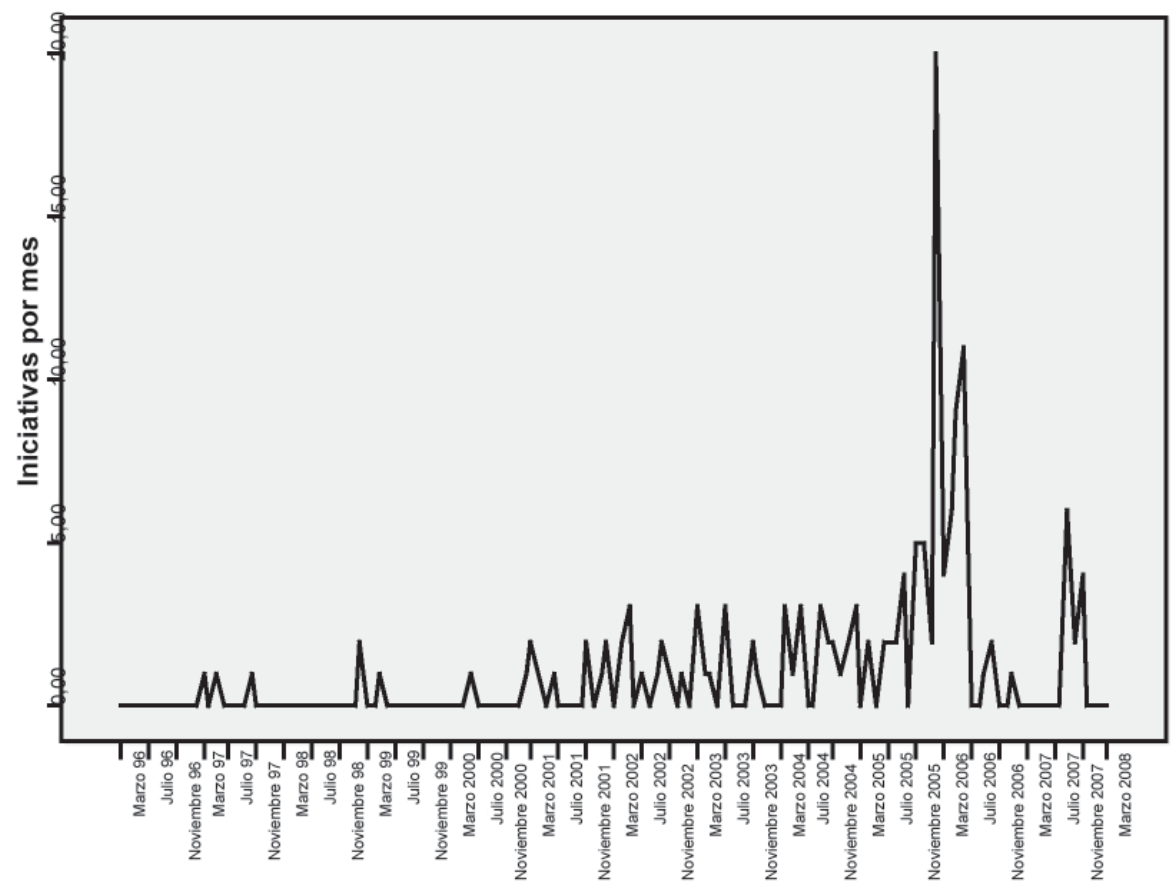

Fuente: elaboración propia a partir del Congreso de los Diputados.

preocupan los desarrollos reglamentarios de la Ley 39/1999 y los permisos que pueden solicitar la ciudadanía española para lograr la conciliación de la vida familiar y laboral. El debate parlamentario de dos propuestas de Ley, la primera del partido socialista sobre los derechos para conciliarla vida familiar y profesional de los trabajadores y trabajadoras, y la segunda del grupo catalán propuesta para la legislación de la licencia de paternidad en caso de parto, adopción o el cuidado de crianza acaparan también la atención de los diputados (13 de mayo 2003).

Pero sobre todo es importante que en la séptima legislatura la Mesa del Congreso de los Diputados, en su reunión de 1 de junio de 2004, acuerda admitir a trámite y remitir a la Comisión de Trabajo y Asuntos Sociales la solicitud del Grupo Parlamentario Popular en el Congreso, relativa a la creación de una Subcomisión para la emisión de un informe que analice la ordenación y adecuación del tiempo de trabajo, la flexibilidad horaria y de la jornada, 


\section{Gráfico 2.}

Tasa de iniciativas sobre conciliación por legislatura (VI, VII y VIII)

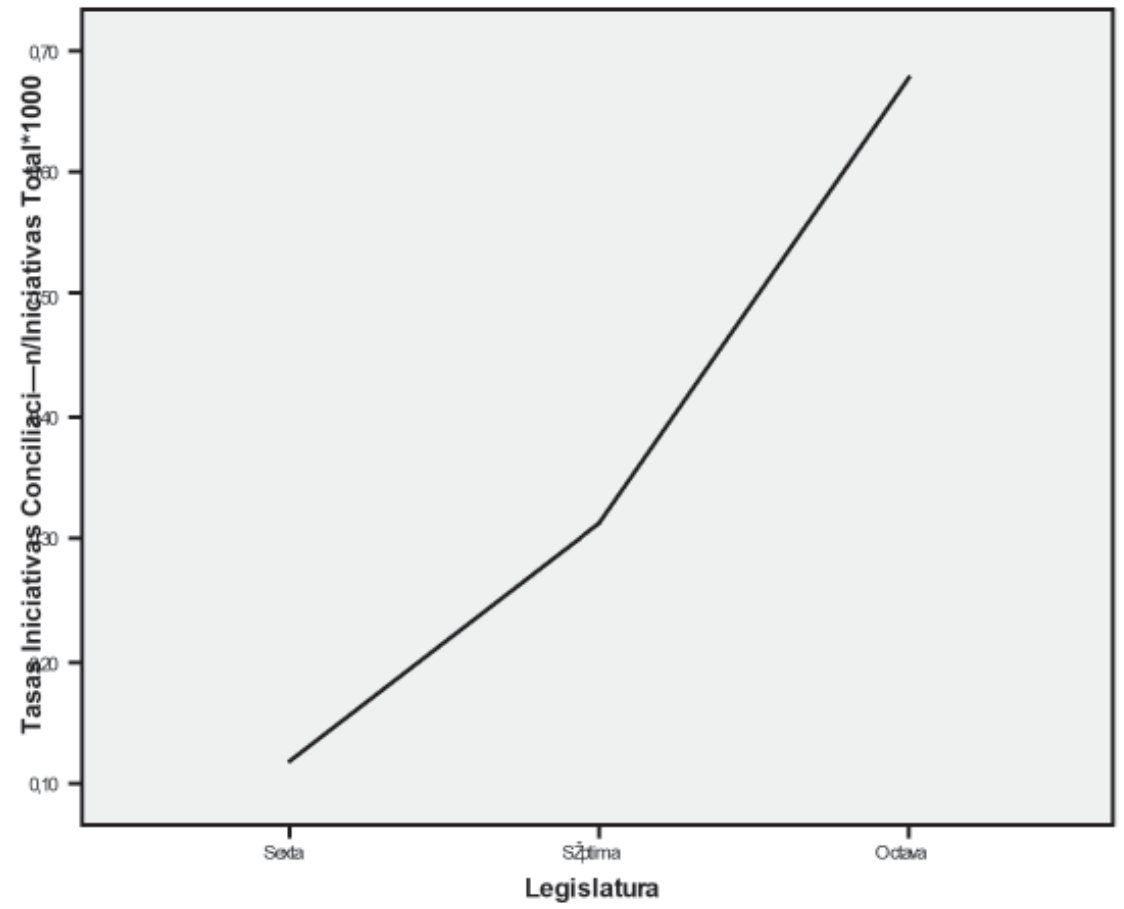

Fuente: Elaboración propia a partir del Congreso de los Diputados.

así como de las posibilidades de adecuación del horario laboral a la conciliación de la vida laboral y profesional (BOCG D-29, de 4-06-04). Entre otras cuestiones se establece que la Subcomisión puede recabar la comparecencia de miembros de las Administraciones Públicas, así como la de autoridades, funcionarios públicos, interlocutores sociales, y cuantas personas y organizaciones resulten competentes en la materia. El plazo para la conclusión del trabajo se establece hasta el 31 de octubre de 2006.

Pues bien, entre el 23 de noviembre de 2005 y el 31 de octubre de ese año, ya con el Partido Socialista en el Gobierno, se producen las comparecencias de 37 expertos y expertas sobre la adecuación de los horarios, que dan cuenta del interés por el tema en el debate parlamentario. 


\section{Gráfico 3.}

Tasa de iniciativas sobre conciliación por años

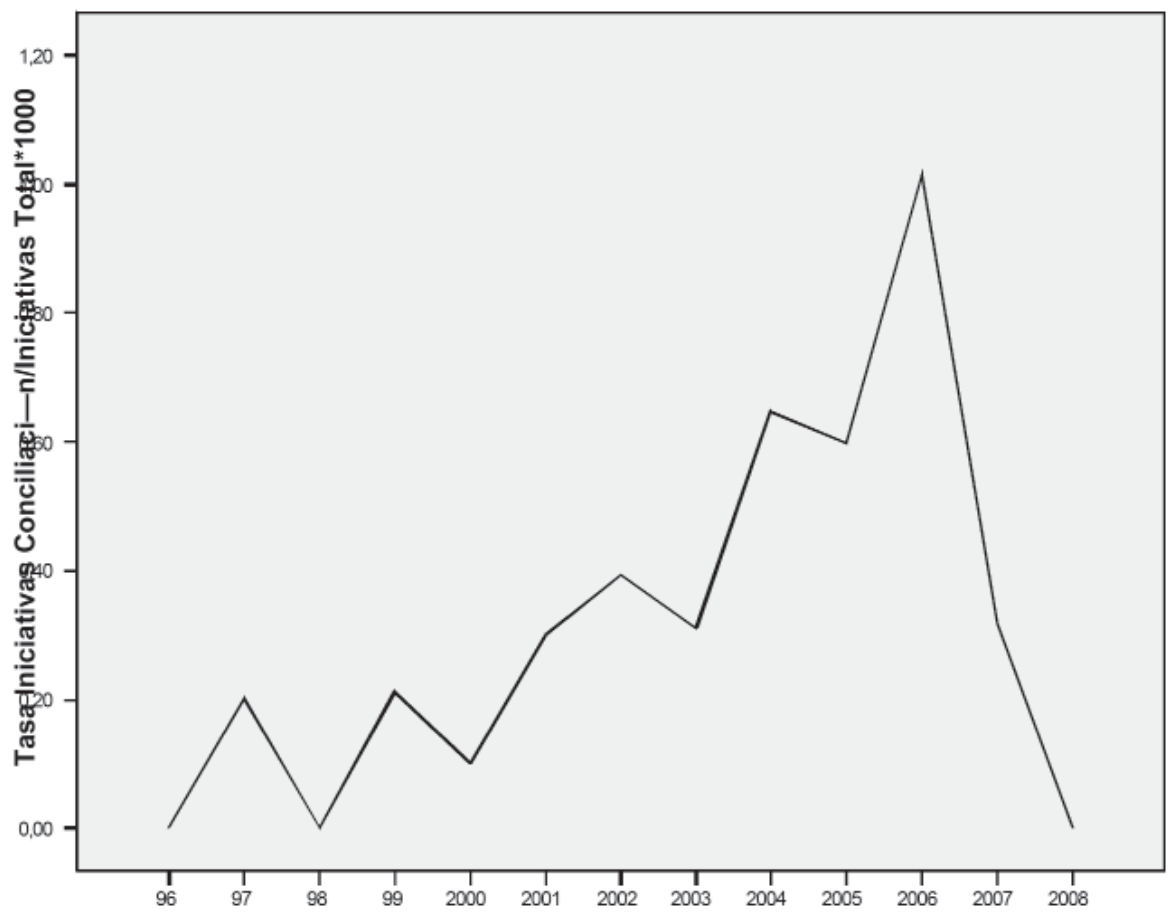

Fuente: Elaboración propia a partir del Congreso de los Diputados.

En estas comparecencias se ponen de manifiesto la disputa sobre nociones a cerca del tiempo «de trabajo, personal y familiar» y sobre «flexibilidad»y «conciliación». Las discusiones, extensas, desbordan el tema inicial propuesto, la regulación de horarios, y terminan tratando cuestiones relacionadas con la igualdad de oportunidades. Las conclusiones de la subcomisión son publicadas en $2007^{6}$ y en ellas el grupo de trabajo expresa que la conciliación no puede producirse hasta que tenga lugar un profundo cambio social que tiene que ver con la erradicación de la mentalidad de la «ayuda», en detrimento de la mentalidad de la «corresponsabilidad». Esta declaración es importante

6. http://www.congreso.es/public_oficiales/L8/CONG/BOCG/D/D_539.PDF, consultado el 9/8/2011 
Gráfico 4.

Presencia del tema por meses. VI, VII, VIII legislatura

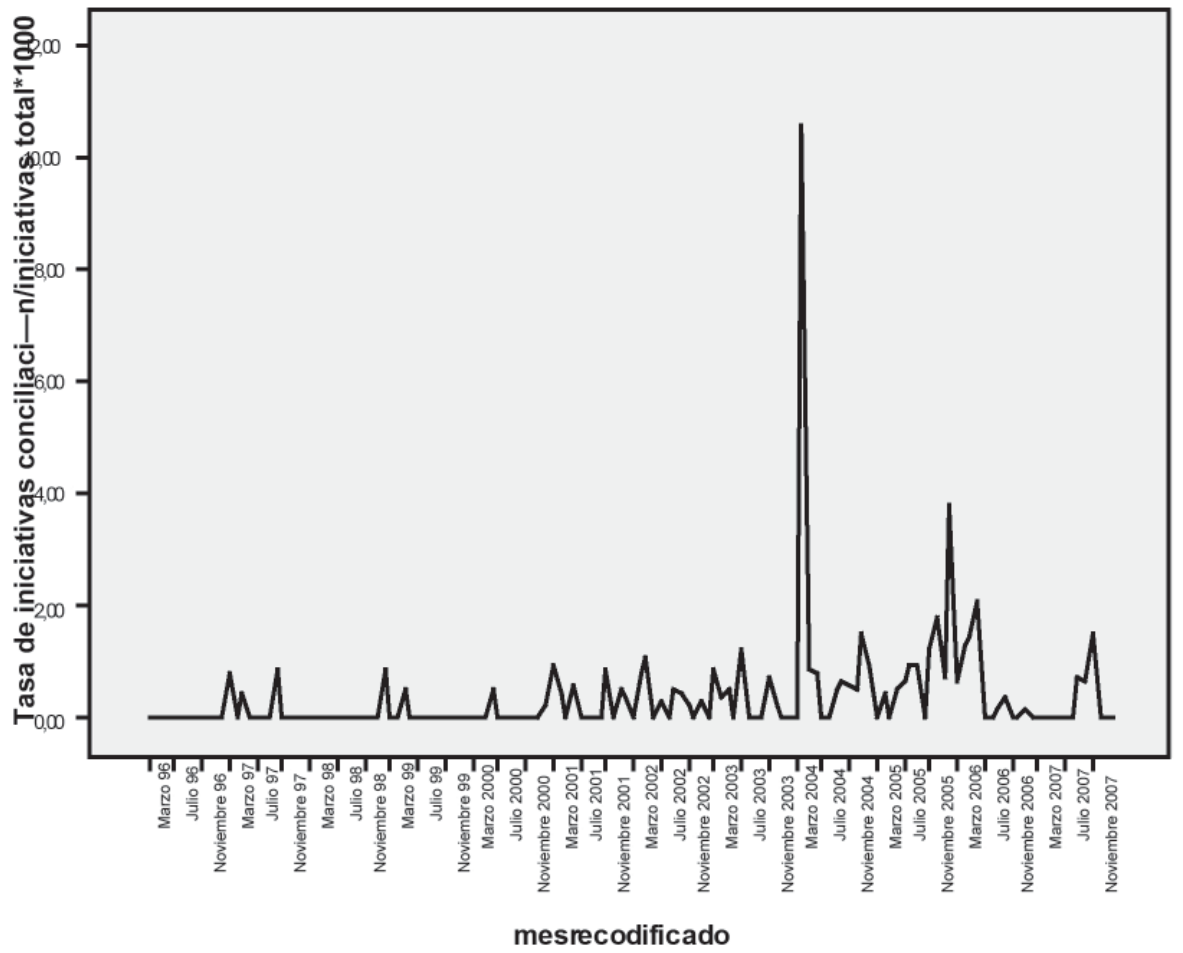

Fuente: Elaboración propia a partir del Congreso de los Diputados.

porque implica el inicio de un giro en el planteamiento del tema. No obstante, el documento finaliza con la petición al Gobierno de que presente un plan de medidas ante el Congreso de los Diputados y con la recomendación de que estas medidas no pueden aplicarse desde una perspectiva neutra, porque afectan fundamentalmente a las mujeres.

Al margen de la discusión sobre la adecuación de horarios en este subcomisión, otras cuestiones que acaparan la atención de los diputados y diputadas son: el control de la aplicación del Plan Concilia (aprobado y publicado en el BOE del 16 de diciembre de 2005, vigente desde 1 de enero de 2006) por parte de la oposición (desde octubre de 2006 y 2007); así como los convenios suscritos entre el Ministerio de Trabajo y Asuntos sociales con distintas CC.AA, para la conciliación a raíz de la aprobación de la Ley Orgánica 3/2007, 
de 22 de marzo, para la igualdad efectiva de mujeres y hombres. Se inquiere también por los presupuestos ejecutados para la conciliación.

\subsection{Las intervenciones parlamentarias sobre conciliación}

El 57\% de las intervenciones parlamentarias sobre conciliación familiar y laboral, durante los años estudiados corresponde a la función de control ${ }^{7}$, el $33,6 \%$ a la orientación política y el 9,4\% pertenecen a la función legislativa.

Los datos se relacionan con el hecho de que el $51,7 \%$ de las menciones se hayan realizado en preguntas escritas dirigidas al Gobierno vigente en cada caso y el 4,7\% preguntas orales al Gobierno en Pleno (2,7\%) o en Comisión (2\%). En segundo lugar, pero con una diferencia porcentual importante, se solicitaron comparecencias en Comisión $(24,2 \%)$, bien de otras personalidades $(19,5 \%)$ o de autoridades y funcionarios $(4,7 \%)$. Las proposiciones de ley $(6 \%)$, y de las no de ley, ante el Pleno o Comisión, fueron el siguiente tipo de iniciativas. Apenas hubo solicitudes de informes y comparecencias del Gobierno.

Dado que muchas de las menciones a la conciliación fueron en preguntas escritas al Gobierno, las iniciativas no se tramitaron en sesiones parlamentarias $(51,7 \%)$. De las que sí se tramitaron, el $13,4 \%^{8}$ pasó por Pleno y el 33,6\% en Comisión. Entre las iniciativas debatidas en Comisión, la mayoría se discutieron en la de Trabajo y Asuntos Sociales $(n=40) ; 6$ en la Comisión Mixta de Derechos de la Mujer; 2 en la Comisión de Política Social y Empleo y 1 en la Comisión de Justicia y otra en la de Defensa.

Muchas de las intervenciones en las que se mencionó la conciliación no llevaron a una toma de decisiones con impacto en las políticas (96,3\%). La mayoría fueron tramitadas sin acuerdo o decisión (76,5\%) o caducaron (8\%).

\subsection{Promotores y promotoras de las intervenciones parlamentarias sobre conciliación}

En un 35\% de las ocasiones, las intervenciones relacionadas con la conciliación fueron promovidas por diputadas ${ }^{9}$, frente a un $22 \%$ originada por hombres y un $42 \%$ en las que no se pudo identificar el sexo del autor. Pese a la mayor presencia global femenina, sólo en la VII legislatura hubo más probabilidad de plantear el tema siendo mujer, (OR = 1,6; IC del 95\%, 1-2,5). Sin

7. Muchas menos que en caso de la inmigración (94\%) y en el caso de violencia de género (93\%).

8. Frente al $33 \%$ de violencia y el $86 \%$ de inmigración

9. Frente al $60 \%$ en el caso de violencia 
embargo, se observó que en la realización de la función de control -iniciativa parlamentaria más frecuente-, los hombres tuvieron una probabilidad mayor de hacer preguntas sobre el tema (OR = 11,6; IC del 95\%, 2,8-46).

El grupo parlamentario popular presentó la mayoría de intervenciones (40\%) seguido de la Comisión de Trabajo y Asuntos Sociales (22\%) y del grupo socialista (19,5\%). Izquierda Unida aparece en cuarto lugar $(7,4 \%)$, seguido de Esquerra Republicana (2,7\%) y del grupo catalán (2\%). De hecho, la conciliación ha sido un concepto más vinculado al grupo popular que al socialista. La probabilidad de realizar iniciativas desde la formación que estaba en el Gobierno fue cuatro veces mayor en el caso de la sexta legislatura $(\mathrm{OR}=4,6$; IC del 95\%, 1,2-10,5) y casi tres veces mayor en la séptima $(\mathrm{OR}=$ 2,8; IC del 95\%, 1,3-6,3), mientras que en la octava, la probabilidad revierte, y es ligeramente mayor en la oposición ( $\mathrm{OR}=1,3$; IC del 95\%, 1,8-1,6). Esta idea se corrobora al hacer los «odds ratio» entre la función parlamentaria y el partido político: hay una relación entre pertenecer al Partido Popular y ejercer la función de control (OR = 3,8; IC del 95\%, 2,1-6,9) y legislativa, mientras la labor del PSOE en el tema de conciliación ha sido únicamente de control (OR $=6,5 ;$ IC del 95\%, 2-20)

\subsection{Temas}

Finalmente, en cuanto a los aspectos tratados en las iniciativas parlamentarias del Congreso, quitadas las cuestiones varias y sobre aspectos puntuales (como por ejemplo la investigación sobre los informes relativos a la conciliación en la Guardia Civil -agrupado todo bajo otros-), el punto más importante y que aparece es el de la regulación de los horarios (28\% iniciativas), en segundo lugar, las transferencia económica y la creación de diversos servicios públicos (10,7\% respectivamente) y, finalmente, la petición de estadísticas y datos (ver tabla 1: temas más importantes tratados en las iniciativas. Años 1996-2008).

En este sentido, hay una diferencia significativa entre el enfoque dado al tema por el PP (centrado en un $27 \%$ en la transferencia económica) y el proporcionado por el PSOE (más ubicado en la creación de servicios públicos, 34,5\%). (Ver tabla 2: Contingencia autor/tema. Años 1996-2008).

Por otra parte, también se aprecian diferencias estadísticamente significativas en el tratamiento de distintos aspectos de la conciliación en virtud el sexo $\left(X^{2}: 39,4 ; p<000\right)$. Así, los hombres concentran más porcentaje de intervenciones sobre horarios, mientras que las mujeres han diversificado su interés por las estadísticas, la transferencia económica y la creación de servicios públicos. 


\section{Tabla 1.}

Temas más importantes tratados en las iniciativas. Años 1996-2008

\begin{tabular}{|l|l|c|c|}
\hline \multicolumn{2}{|l|}{} & FRECUENCIA & PORCENTAJE \\
\hline Válidos & Transferencia Económica & 16 & 10,7 \\
\hline & Horarios & 42 & 28,2 \\
\hline & Servicios públicos & 16 & 10,7 \\
\hline & Estadísticas/datos & 13 & 8,7 \\
\hline & Otros & 62 & 41,6 \\
\hline & Total & 149 & 100 \\
\hline
\end{tabular}

Fuente: elaboración propia a partir del Congreso de los Diputados.

Tabla 2.

Contingencia autor/tema. Años 1996-2008

\begin{tabular}{|c|c|c|c|c|c|c|c|c|}
\hline \multicolumn{2}{|c|}{} & & \multicolumn{5}{|c|}{ TEMA } & TOTAL \\
\hline \multicolumn{2}{|c|}{} & $\begin{array}{c}\text { Trans- } \\
\text { ferencia } \\
\text { econó- } \\
\text { mica }\end{array}$ & Horarios & $\begin{array}{c}\text { Servicios } \\
\text { públicos }\end{array}$ & $\begin{array}{c}\text { Estadís- } \\
\text { ticas/ } \\
\text { datos }\end{array}$ & Otros & $\begin{array}{c}\text { Trans- } \\
\text { ferencia } \\
\text { econó- } \\
\text { mica }\end{array}$ \\
\hline Autor & PSOE & Recuento & 0 & 2 & 10 & 7 & 10 & 29 \\
\hline & & $\begin{array}{c}\% \text { dentro } \\
\text { de autor }\end{array}$ & 0 & 6,9 & 34,5 & 24,1 & 34,5 & \\
\hline & PP & Recuento & 16 & 3 & 4 & 3 & 35 & 61 \\
\hline & $\begin{array}{c}\% \text { dentro } \\
\text { de autor }\end{array}$ & 26,2 & 4,9 & 6,6 & 4,9 & 57,4 & \\
\hline \multicolumn{2}{|c|}{ TOTAL } & Recuento & 16 & 5 & 14 & 10 & 45 & 90 \\
\hline
\end{tabular}

Fuente: elaboración propia a partir del Congreso de los Diputados.

Los porcentajes y los totales se basan en los encuestados.a Agrupación de dicotomías.

Tabulado el valor 1.

\section{Discusión}

La conciliación familiar y laboral entra en la agenda parlamentaria española en 1999, ante la necesidad de aprobación de una Ley para la adaptación de la normativa Europea bajo pena de sanción. La irrupción del tema en nuestro país es, políticamente hablando, un tanto abrupta. Europa impone regular un asunto casi ignoto en España, presente sin embargo, con fuerza, en la agenda internacional desde el 95, cuando la Conferencia de Pekín plantea la necesidad de dar respuesta a un «problema social en desarrollo». La conciliación de 
los trabajadores y trabajadoras se define desde las instancias supranacionales como un obstáculo para la igualdad, entendida ésta en el terreno de la incorporación plena al mercado. Y es en estos términos en los que la Ley 39/1999 pretende dar una solución.

Así, en la VI legislatura, el Gobierno del PP se ve obligado a introducir nuevos permisos parentales y a modificar la legislación laboral, sin un debate previo que genere la fecundidad de ideas para tener fuerza política. Ante un escenario en el que la importancia de la cuestión no está asimilada todavía por la opinión pública ni, probablemente, por la mayoría política, no sorprende la lentitud de las iniciativas parlamentarias (Guirao, 2010: 178).

Una década después, cuando acaba el periodo analizado (marzo de 2008), España presenta un marco jurídico, si no puntero, si prolífico sobre este tema. Como muestran los datos, no es hasta 2001, cuando se recoge el efecto de la incorporación en campaña electoral de la importancia de los programas de conciliación laboral y familiar, cuando el tema empieza a cobrar fuerza.

La construcción parlamentaria del asunto se inicia por el grupo Popular (con un $40 \%$ de las iniciativas), pero despega en el marco social creado por el PSOE cuando experimenta una evolución cualitativa. La conciliación nace, impelida por Europa, como un «artefacto» político para la aceleración de la incorporación de la mujer a un mercado de trabajo cada vez más ávido de mano de obra flexible (da cuenta de ello su tratamiento habitual la Comisión de Trabajo y puntual en la de Igualdad). Así, las normas promueven medidas para compatibilizar el trabajo productivo y reproductivo de las mujeres. El hecho de que también los datos de incorporación de las mujeres el mercado laboral acaparen las preguntas del control a los Gobierno refuerza la tesis de que, pese a ser presentadas como «conciliación», muchas acciones de corte laboral son persecutorias del pleno empleo (no de la erradicación del conflicto).

Esta perspectiva inicial se matiza a finales de 2005, en la VIII Legislatura (entre 2004 y 2008), cuando el tema se vincula a la igualdad de oportunidades, y no sólo laborales, entre sexos. En el escenario de la «normalización» pública de este concepto, a partir de la visión de los expertos, se empiezan a repensar los usos del tiempo (público, doméstico y personal) y las cargas del trabajo. Se aborda así la necesidad de iniciar políticas sobre lo que históricamente estaba relegado al ámbito de lo privado (organización doméstica del cuidado y de la reproducción), como ejemplifica el auge del término «corresponsabilidad» o la ulterior promulgación de la Ley de Dependencia. En cualquier caso, parlamentariamente hablando, la conciliación se sigue vinculando con el empoderamiento para el trabajo y no con la salud mental y el bienestar de la ciudadanía. 
Es en el marco de la igualdad entre sexos, en el que se integra este tema a partir del Gobierno del PSOE. No obstante, si comparamos y analizamos los debates sostenidos sobre otras cuestiones -como la violencia machista o la inmigración-, se pone de manifiesto que el asunto es mucho menos polémico (el porcentaje de intervenciones de control es menor) y mucho menos impulsado por las mujeres diputadas que los anteriores.

Con la perspectiva de más de una década, se observa, por una parte, que el tema, con un gran eco mediático ha tenido una importancia parlamentaria (12 iniciativas por año) muy inferior a otras cuestiones objeto de políticas públicas como la inmigración (51 iniciativas por año) o la violencia machista (22 iniciativas por año). Por otro lado, se detectan diferencias de planteamientos entre formaciones. El Partido Popular (que presenta el 40\% de las iniciativas) habla reiteradamente de la transferencia de recursos a las familias y de la regulación y flexibilización de los horarios laborales (visión con la que, por sexo, parecen coincidir los hombres). Por su parte, el PSOE, con un discurso en el que el concepto de la conciliación está mucho menos presente, centra más su enfoque parlamentario en la instauración de distintos servicios públicos para las tareas de cuidado.

Las discrepancias retóricas se plasman en las decisiones políticas. De la mano del PP se promulga Ley 39/1999, de 5 de noviembre, para promover la conciliación de la vida familiar y laboral de las personas trabajadoras, que propone la provisión de medidas de flexibilidad en el mundo del trabajo y los incentivos por nacimiento de hijos, dirigidas mayoritariamente a las féminas. De la mano del PSOE, La Ley para la promoción de la autonomía personal y atención a las personas en situación de dependencia», en 2006, que pretende «corresponsabilizar» al estado de las tareas de cuidado de algunos ciudadanos, (aunque su puesta en marcha ha sido criticada por fomentar la «prestación económica para cuidados en el entorno familiar» frente a la inversión en servicios de cuidado y por la feminización de los cuidados en la que ha resultado). Finalmente, y también bajo el mandato de este partido, se promulga Ley Orgánica 3/2007, para la igualdad efectiva de mujeres y hombres, que empieza a reconocer «el papel masculino dentro de la conciliación y su obvia importancia dentro de la familia» (Chinchilla y León 2011: 51) y la Ley 9/2009, de 6 de octubre, que amplía el permiso de paternidad.

A la luz de los datos y de la revisión del ciclo de interés político por el problema, no es arriesgado afirmar que, en cierta medida, a finales de la VIII legislatura y con el cambio de enfoque, el concepto de «conciliación» quedaba herido de muerte, al haberse incorporado otros vocablos, como 
corresponsabilidad, o haber sido eclipsado por otros temas relacionados con el género como la violencia.

En un contexto económico y laboral internacional y nacional completamente distinto al de 2008, cabe ahora volverse a plantear hasta qué punto la noción, el debate y las políticas (inicialmente ideadas para la incorporación plena de la mujer al mercado laboral) van a ser, parlamentariamente hablando, reconsiderados.

No queremos finalizar el trabajo sin hacer referencia a algunas limitaciones. La primera se refiere a la circunscripción del análisis a la Cámara de los Diputados. Dado el objeto de este estudio, y considerando el carácter de segunda cámara, no hemos tenido en cuenta en el trabajo los datos referentes al Senado, ni los relativos a las Asambleas Parlamentarias de las comunidades autónomas. Habida cuenta de la transferencia de competencias en muchos aspectos resulta relevante comparar el tratamiento del tema en estos ámbitos para una ulterior investigación.

Por otra parte, cabría matizar la bipolarización (PP frente a PSOE) cuando ha habido claramente gobiernos minoritarios y, por lo tanto, sometidos a la influencia de otros grupos - esencialmente CiU y Ezquerra-. En este sentido, sería interesante para un estudio posterior con un enfoque cualitativo no pretendido, ver qué iniciativas tuvieron realmente éxito y cuáles no y por qué causa.

\section{Referencias}

Agudelo-Suarez, A., Gil-González, D., Vives-Cases, C., Ronda-Pérez, E. (2009). «The Parliamentary debate on immigration and health in Spain». Revista Salud Pública [online] 11 (4), pp. 503-513.

BALLESTER, M.A. (2011). «Conciliación y corresponsabilidad en la Unión Europea» en Cabeza, J. (ed.). Conciliación de la vida familiar y laboral y corresponsabilidad entre sexos. Valencia: Tirant lo Blanc, pp. 17-49.

Bustelo M., Lombardo E. (eds.) (2007). Políticas de igualdad en España y en Europa. Madrid: Cátedra.

Bustelo, M., Peterson E. (2005). «Conciliación y (des)igualdad. Una mirada debajo de la alfombra de las políticas de igualdad entre mujeres y hombres». Revista de Desarrollo y Educación Popular. Hombres y mujeres: coeducación. $\mathrm{N}^{\circ}$ 7, pp. 32-37.

CABEZA, J. (2010). «Conciliación de la vida privada y laboral». Temas laborales. $N^{o} 103$, pp. 45-65. 
Cambronero-Saiz, B., Ruiz-Cantero, M., Vives-Cases, C., Carrasco-Portiño, M. (2007). «Abortion in democratic Spain: the parliamentary political agenda 1979-2004». Reprod Health Matters 15 (29), pp. 85-96.

Chinchilla, N., León, C. (2011). Diez años de conciliación en España. Madrid: Grupo 5.

EFILWC (2006). Reconciliation of work and family life and collective bargaining in the European Union: An analysis of EIRO articles. Fundación Europea para la Mejora de las Condiciones de Vida y de Trabajo. Publicaciones Unión Europea. Disponible en http://www.eurofound.eu.int/pubdocs/ 2006/06/en/1/ ef0606en.pdf. (9/8/2011).

Feliu-Albaladejo, A. (2011). «La publicidad institucional en la arena parlamentaria española». Revista Latina de Comunicación Social. No 6, pp. 454 -481.

FERnÁndeZ, J. A., Tobío, C. (2005). Conciliar las responsabilidades familiares y laborales: políticas y prácticas sociales. Documentos de trabajo 79/2005. Madrid: Fundación Alternativa.

FlaQUER, L. (2000). Las politicas familiares en una perspectiva comparada. Barcelona: Fundación 'la Caixa' (Colección Estudios Sociales, 3)

GUIRAO, C. (2010). La construcción social del problema de la conciliación vida familiar y laboral en España, 1999-2009. Tesis Doctoral. Dirección: Marta Martín Llaguno y Dolores Frutos Balibrea. Universidad de Alicante.

Hochschild, A. (1989). The Second Shift: Working Parents and the Revolution at Home. New York: Viking.

Iglesias de Ussel, J., Meil Landwerlin, G. (2001). La política familiar en España. Barcelona: Ariel.

Ley 39/1999, de 5 de noviembre, para promover la conciliación de la vida familiar y laboral de las personas trabajadoras, BOE 266, de 6 de noviembre de 1999, pp. 38934-38942.

Ley 39/2006, de 14 de diciembre, de promoción de la autonomía personal y atención a las personas en situación de dependencia, BOE 299, de 15 de diciembre de 2006, pp. 44142-44156.

Ley 40/2003, de 18 de noviembre, de protección a las familias numerosas, BOE 277, de 19 de noviembre de 2003, pp. 40845-40852.

Ley 46/2002, de 18 de diciembre, de reforma parcial del impuesto sobre la renta de las personas físicas y por la que se modifican las leyes de los impuestos sobre sociedades y sobre la renta de no residentes, BOE 303, de 19 de diciembre de 2002, pp. 44622- 44662.

Ley 9/2009, de 6 de octubre, de ampliación de la duración del permiso de paternidad en los casos de nacimiento, adopción o acogida, BOE 242, de 7 de octubre de 2009, pp. 84692-84694.

Ley Orgánica 3/2007, de 22 de marzo, para la igualdad efectiva de mujeres y hombres, BOE 71, de 23 de marzo de 2007, pp. 12611-12645. 
Ley Orgánica 3/2007, de 22 de marzo, para la igualdad efectiva de mujeres y hombres. BOE 71, de 23 de marzo de 2007, pp. 12611-12645.

Lombardo, E. (2003). «El mainstreaming de género en la Unión Europea». Aequalitas. Revista Jurídica de Igualdad de Oportunidades entre Mujeres y Hombres, vol. 10-15, pp. 6-11.

Lombardo, E., MeIER, P. (2006). «Gender mainstreaming in the EU. Incorporating a feminist reading?». European Journal of Women's Studies 13 (2), pp. 151-166.

Martín llaguno, M., Beléndez, M., Hernández, A. (2007). La mujer en las agencias de publicidad. Categorías, especializaciones y conflicto trabajo-familia en las agencias españolas. Madrid: Asociación Española de Agencias de Publicidad.

Naciones Unidas (1995). Cuarta Conferencia Mundial sobre la Mujer: Beijing, China.

Astán ChaVes, R., Rodríguez Sosa, V. (2010). "¿Cómo puede afectar la crisis económica a la conciliación?». XII Jornadas de Economía Crítica. Disponible en http://www.ucm.es/info/ec/jec12/programa.html (14/4/2012).

Renton, A., Macintosh, A. (2007). «Computer supported argument maps as a policy memory». Information Society Journal 23 (2), pp. 125-133.

Vives-Cases, C., Gil-GonZÁlez, D., CARRASCo-Portiño, M., Álvarez-DARdet, C. (2006). «La violencia de género en la agenda del Parlamento español (19792004)». Gaceta Sanitaria: Órgano Oficial de la Sociedad Española de Salud Pública y Administración Sanitaria 20(2), pp. 142-8. 